\title{
NEW SYSTEMS FOR THE MANAGEMENT OF DATA. THE CASE STUDY OF THE CHAPTER HOUSE OF SANTA MARIA NOVELLA IN FLORENCE
}

\author{
C. Battini ${ }^{\text {a }}$
}

a DICCA, University of Genoa - carlo.battini@gmail.com

KEY WORDS: Documentation, Image Matching, Laser Scanner, Multiresolution, Virtual Reality, Web, Webgl

\begin{abstract}
:
The technological solutions, made available today, offer opportunities of great interest for the detection in the field of cultural heritage; instrumentation for the survey and advanced multimedia representations for objects of archaeological, artistic, architectural.

Since we changed the type of information, not more interpretation consists of a single piece of data, but from a set of data, must be changed, consequently, also the systems for their management or better, if until now the only situation of interest was that of having to deal with the data representing only the text, data are now made up of sounds, images, video and three-dimensional models. For these reasons and others, we can discuss in continuation, systems and storage structures classics are still not sufficient to manage these new realities.

An architectural structure is a set of three-dimensional components that define spatially a form and a project idea; so that the representation of the architecture may not avail itself simply means two-dimensional graphs to describe it and describe it in all its parts, but need, and it is now possible, systems that provide for the possibility to analyze from multiple points of view the forms that compose it.

The communication media can play a very important role to get directions on the actions of restoration and enhancement of the cultural and environmental heritage. Help can be provided by information and its simulation systems of virtual reality with which, in addition to conveying information, you can view models to better describe the development that the well has had in history.

This research starts from the need to develop new systems of representation and data management. Objective of the project is to increase and share knowledge of architecture and the environment. Dynamic representations, relational databases, devices use data, are the main tools of this research.

The study addressed led to the creation of a system that allows the transmission and analysis of data collected and, at the same time, also creates the possibility of access to users not experts in the field of 3D graphics; this has been made possible through the development the project "3DWS" (3D | WEB | SURVEY).

The project involved the creation of a container structured as a web site, in which were placed the materials collected and processed including sketches, photographs, vector data, three-dimensional models and analysis issues. Within this system, three-dimensional models developed were then used as a communication tool with immediate visual link to sub-pages with a greater degree of definition. It is a simple and intuitive tree structure that allows both the visitor to the specialist scholar to focus on portions too small and detail of the product, without losing sight of the general configuration of departure.

To make feasible a further degree of usability of information, the system has been implemented with image viewers metrics in high definition. The images produced are a fundamental basis, strictly from the point of view of science and technology, for the conservation, management and enhancement of the historical and artistic heritage.

The case study investigated is the Chapter House of Santa Maria Novella in Florence.
\end{abstract}

\section{INTRODUCTION}

The project developed in this research will be a test of the possibility that a management system can provide through use of graphic information, textual, numerical and multimedia resulting from the observations and closely linked to a real object.

The construction of a management system is often the part with which researchers have significant difficulties; with the formation of the database that will collate all the information collected and that will organise the data arising from the state of the art concerning the knowledge of the object under investigation and to form a solid basis for the collection of new data.

The issues arising from this process are mainly due to imperfect synchronization and a lack of understanding between those collecting the information and the researchers who later interpret the data; a problem that often limits the development potential of a specific investigation. In addition, frequently these platforms are designed using special proprietary software developed for other purposes, chosen for their ease of use and availability of documentation offered by the software houses. However, the use of the system cannot be limited by the knowledge of particular applications or by the timing and guidelines that the manufacturer adopts.

The programming structure of the system must, at any time, allow for as many upgrades as are necessitated by the particular methodology that should be applied to the single artefact, or in order to improve the researcher's ability to understand and disseminate the information collected. It is clear, therefore, that the same analysis procedure cannot be applied to each case study, nor can the same representation and cataloguing techniques be used for the data collected.

To avoid encountering the aforementioned problems, we have tried to analyse the process and the steps required for the development and investigation of a cultural heritage. After the retrieval of data to the definition of critical elaborations of a 
particular artefact, there are processes which, if optimised, can save a considerable amount of time and ensure a higher reliability of the results obtained. The computer system must express, in an articulate manner, their capabilities and meet the requirements laid out during the design phase.

As is generally recognized, the management of spatial data, both environmental and architectural, through existing tools of information technology, especially in a system like the one presented, requires the data to be structured in categories of objects, whose instances demonstrate similar properties. Any representation of such architectural objects must be fully defined (shape, description, location in space), using its spatial component, alphanumeric and topological.

Among the main considerations, of course, is the representation at different levels of detail and the properties of objects. In addition, the database should support a variety of queries according to the requests that the user formulates at that precise moment.

As a case study to test the system we considered the importance of the Spanish Chapel in the Church of Santa Maria Novella. This room's vaulted surfaces and rich frescoes make it particularly interesting for testing the system adopted. Its paintings were used to test the technology for displaying highdefinition images using the metrics tool Modus Explorer produced by Cultura Nuova, a company specializing in the field of cultural heritage.

The so-called Spanish Chapel is the old chapter house of Florence's Santa Maria Novella church. Famous for the frescoes of Andrea Bonaiuto (1365-1367), the church assumed its present name in the sixteenth century when it was used by the Spanish court in the wake of Eleonora of Toledo, who married Cosimo I de 'Medici. It can be accessed from the Green Cloister, and is now part of the Museum of Santa Maria Novella.

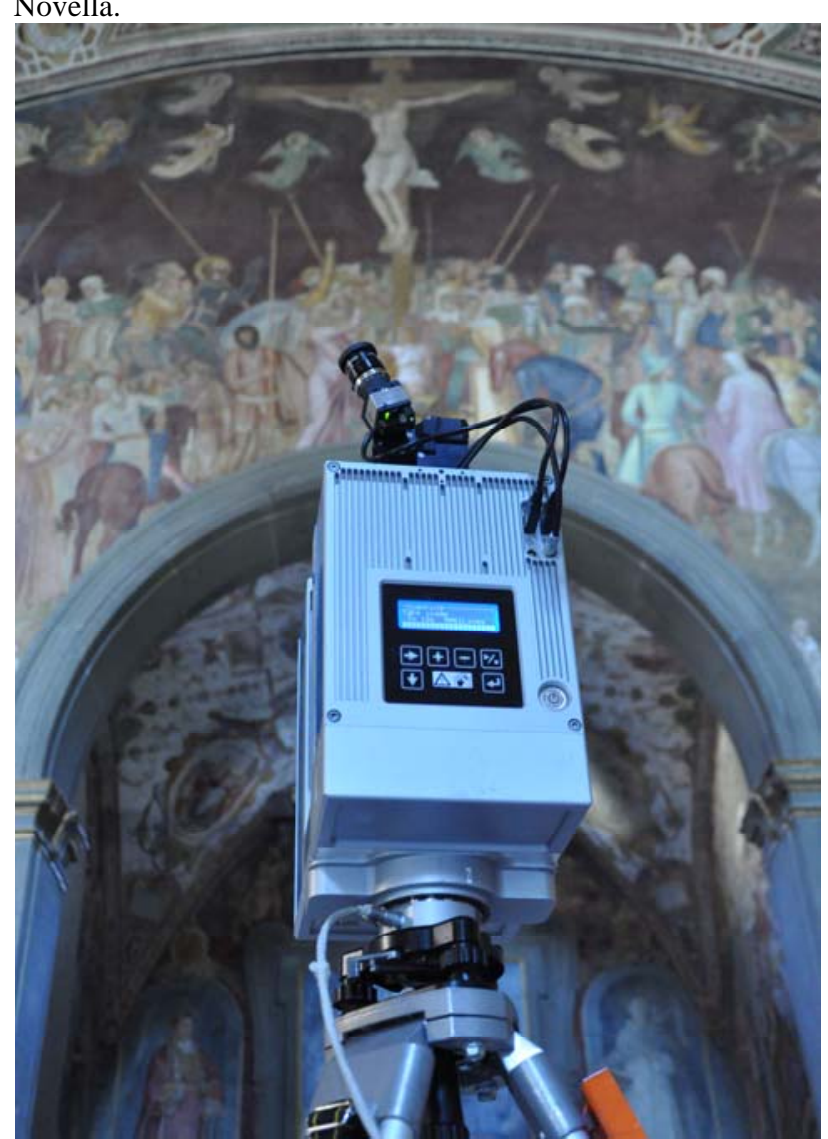

Figure 1. Laser scanner Z+F Imager 5006i

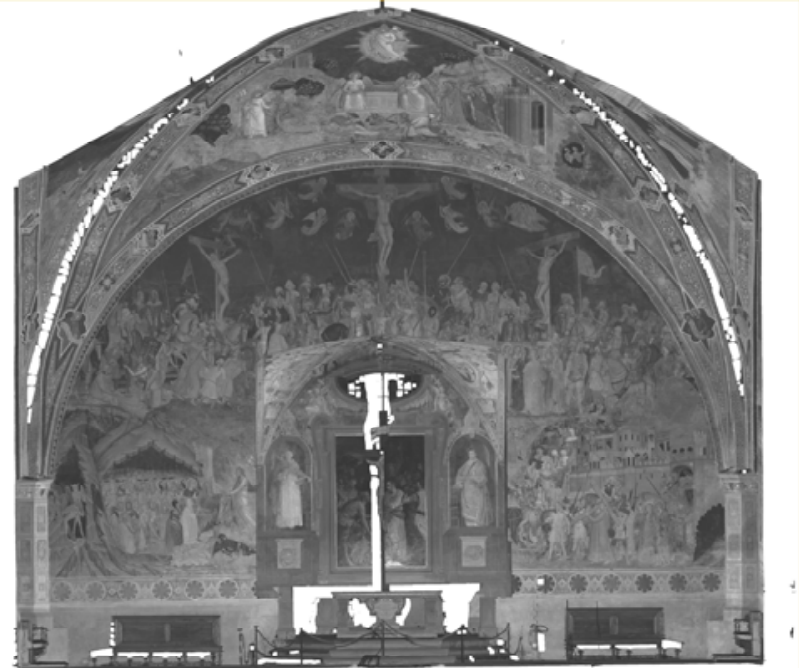

Figure 2. Image of point cloud acquired in grey scale

The theme of the frescoes is the exaltation of the Dominican order and, in particular, the fight against heresy for the salvation of Christianity. In the four sails are depicted the Balance of St. Peter the Apostle, the Resurrection, the Ascension and Pentecost.

The metric survey was carried out using a laser scanner phase shift $\mathrm{Z}+\mathrm{F}$ Imager 5006i which allowed us to acquire a dense point cloud of the entire room. Photographic images were also collected which allowed the adding of colour to the point cloud. Further photographs were taken with a Nikon D5000 camera to create a high-resolution mosaic of painted surfaces, material which was then processed by the Modus Explorer software.

\section{THE MANAGEMENT SYSTEM}

Keeping in mind the considerations above, the management system was built from the design of the graphic template. This is the base, the interface through which the user may make use of the system's potential quickly and intuitively. For this reason, the screen that the operator sees is divided into parts, in which there are certain functions and menus that change according to the screen chosen. At the top of the screen there is a header, common to all the pages, that contains the name and a representative image of the system. Similarly, a common footer displays general information regarding the system. The remainder is divided into three parts, each of which comprises sets of data classed according to type and characteristics of use. On the left is the menu that allows the user to choose the required system; in the top right the title of the current sheet is displayed and, when necessary, special links that allow user interaction with the system also appear. Finally, in the bottomright corner, are the screens for data entry and for searching for data already in the database.

The structure comprises of four main sections: Home, Case Studies, FAQs and Contact Information. The Home page includes a description of the operation of the system, highlighting the features and benefits.

The FAQ section contains the definition of various operational procedures and the solution to problems that the user may find in the use the proposed package.

The contact page shows information concerning all the researchers and institutions which have aided in various stages of the system's development, or have carried out the surveys. 


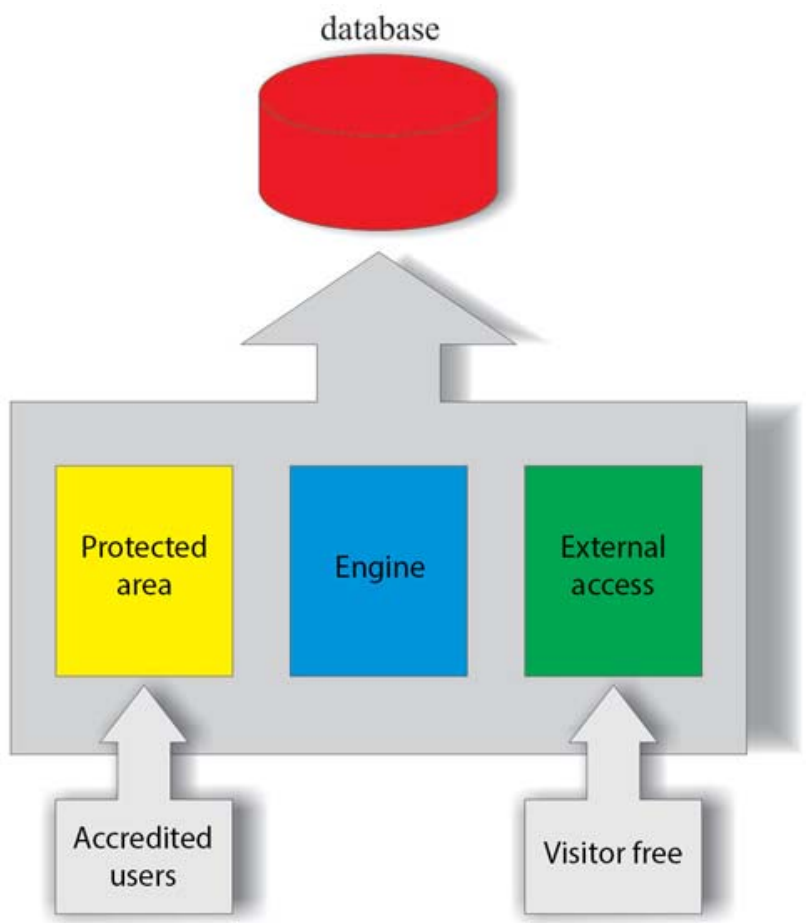

Figure 3. Schematic of the permit system adopted. Enabled users have access to protected areas.

The "Case Studies" section contains all the categories and operations necessary for the cataloguing and viewing of the information collected or processed. Its sub-categories are made up of data and processes that, following the selection of the case study to be analysed, allow the entering of queries and the updating of the database.

The "Case Study" screen is designed to contain the basic information to describe the object studied. The following fields are available to the operator:

- The title;

- A brief description;

- A field within which you can enter a fuller description;

- GPS coordinates of the complex under analysis;

- Fields to insert three representative images;

- A field to insert a schematic representative model which not only allows the user to understand the geometry of the area under analysis, but also to find the images relating to the category "data".

In particular, this last option makes it possible to generate a navigable X3D model containing information that the user can enter into the data entry category, making the research easier and more interactive, and facilitating the identification of the parties concerned in the studies.

In addition, in the on-screen text, you can insert an attachment containing scans of the texts analysed. In order to make the navigation and use of the system easier, it is designed to not to restrict the use of particular formats of attachments, therefore allowing the user to share any kind of file.

The surveys screen includes fields that allow the insertion of the vector information and graphics related to the adopted methodology. In this way you can see the surveys carried out, download the source data and monographs, and then edit the survey in order to increase the range or enrich particular aspects, as in the case of measurements taken for the study.

Obviously, the data in the fields displaying three-dimensional information is very limited, useful for a global representation of the architecture investigated, but not a reliable data source for those wishing to analyse the item with the same level of accuracy as the instrument originally used. Indeed, as previously described, the three-dimensional description of the geometric information gathered during the survey is aimed at a representation of the shapes that constitute the item analysed by aiding the understanding of how the architecture and its surroundings develop. In order to perform with more speed and accuracy fields have been inserted within which the user can attach the source of the findings. Finally, the image screens include fields dedicated to the description and interpretation of the photographed object, as well as its geometric coordinates, taken from the schematic three-dimensional model included in the "Case Studies" screen.

Similarly, the data processing section is divided into categories to better ensure the pooling of information and to make it more easily accessible.

The system examined was therefore created by designing a series of data insertion fields able to acquire the information and save it in the database. In addition, to cater to the operator's needs, the system interface was designed to replicate the layout of paper records. The formation of a database from paper records provided an opportunity to streamline the data entry process. Some fields are simply multiple-choice in order to facilitate this process. The data collected is stored automatically within the SQL database when the user clicks "send" at the bottom of the sheet.

\section{MODUS EXPLORER}

One of the updates made to the system is the use of highresolution images. The high-resolution photographs have been the basis for the construction of HD metric pyramidal images for study over the Internet. To implement this technology required Modus Explorer, the system produced by the New Culture study. This is an instrument that uses multiple information collected within the database. The system is able to show layers of distinct and overlapping data contained in the archive with varying levels of magnification, whilst maintaining a relative scale to the real work.
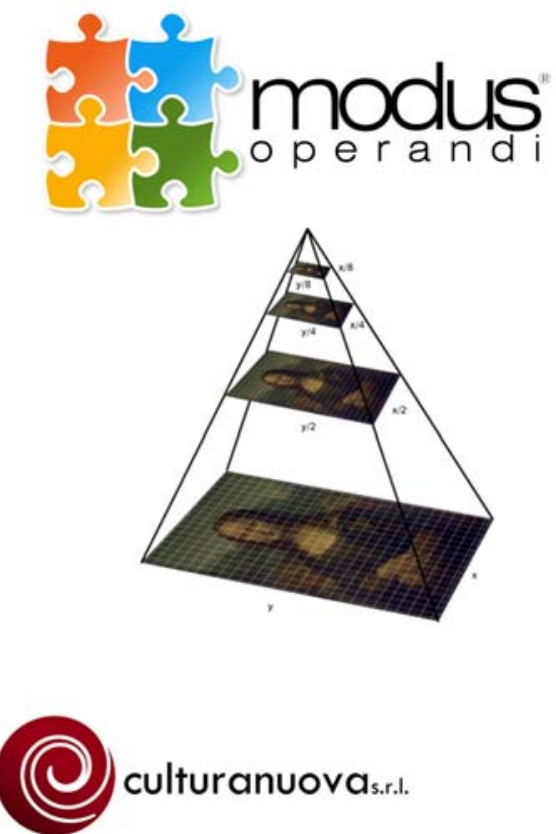

Figure 4. Modus Explorer is a utility for Modus Operandi, management system produced by Cultura Nuova s.r.l. 
Using this system, it is possible to export certain information such as sequences of images, texts with details of the painted surface and thematic maps containing information which would otherwise be summarized in a simpler, less accurate way. By logging in with a username an password, it is also possible to access restricted sections in which details can be altered.

Among the most important features of this system is the provision of graphical tools designed also for use in touch screen terminals.

\section{WEBGL AND THREE-DIMENSIONAL MODELS}

Three-dimensional visualization has now become essential for the representation of this type of information. Ever more efficient systems are used to display data in space, location and the spatial representation of information that until a few years ago were restricted to two-dimensional images. For this reason, the research project has developed a particular syntax and procedure capable of displaying three-dimensional models by utilising the information stored in the database.

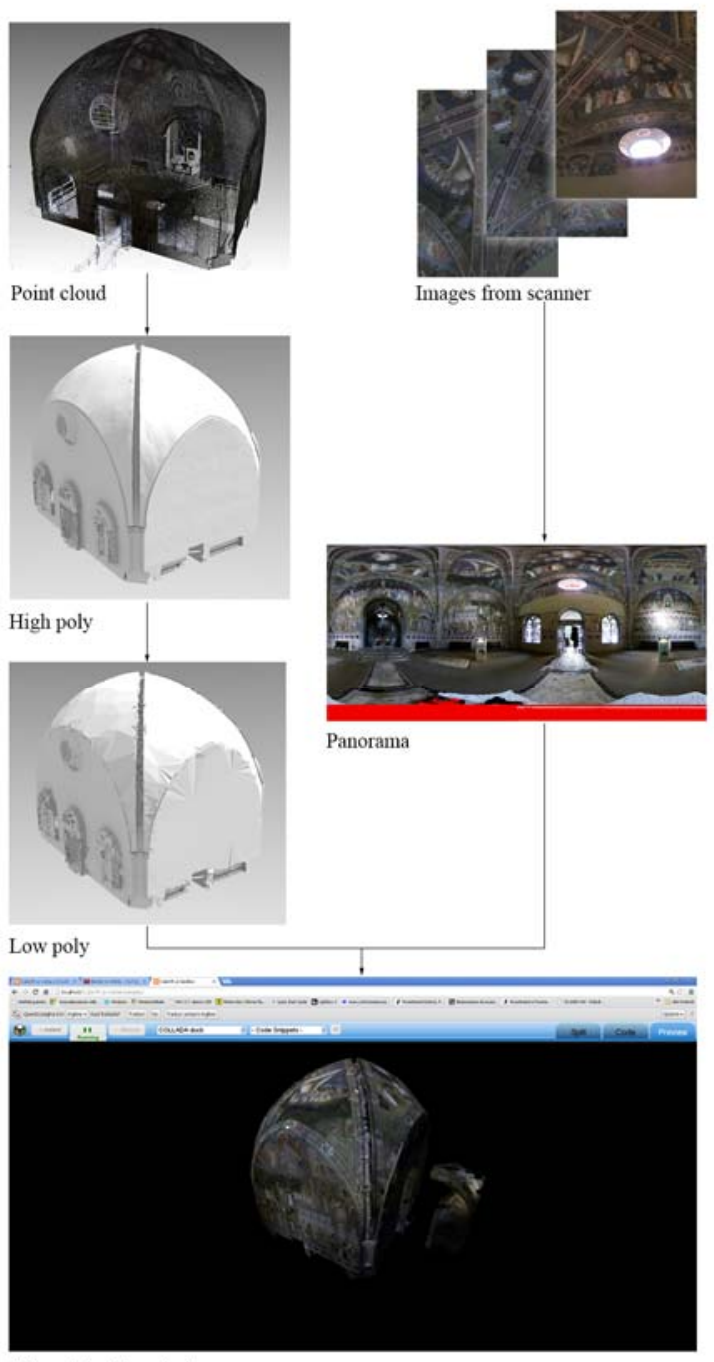

3D model with webgl

Figure 5. Schema to trasform point cloud in webgl models
Data used in dynamic pages can display not only the spatial geometry of the asset being analysed, but also the placement of particular points that allow access to detailed files, images, text and other information.

A trial product, WebGL (Web Graphics Library, a free JavaScript API), has been linked to this display system, to produce interactive 3D graphics and 2D graphics via any compatible web browser, without the use of plug-ins.

In this case we have inserted the point cloud of the Chapter House to better display its geometry. The advantage of this technology, currently under development, is that any user can navigate through three-dimensional models or point clouds without the need for special software or hardware.

\section{CONCLUSIONS}

The proposed system is intended to be an example of what you can do with communication technologies. The goal is to create a web-based system for information sharing and visualization of data collected during a survey of cultural heritage. To address this issue a system was created based on platforms that can be developed to implement new technologies. The system should be considered as a prototype which remains open to new methods of evolution and interpretation. In particular, the way in which three-dimensional images interact with the information database should be further developed. Finally, the display of high resolution images should be implemented to support the surveys and their interpretation.

\section{REFERENCES}

Guidi G., Russo M., Beraldin J., 2010. Acquisizione 3D e modellazione poligonale. McGraw-Hill, Milano

Razionale A. V., Plescia G., Sagone L., 2012. In Rossi M., Siniscalco A. (a cura di), Colore e Colorimetria. Contributi Multidisciplinari. Vol. VIII A, Maggioli Editore, Santarcangelo di Romagna (RN), pp. 23-30

Atalan Cayirezmez N., 2007. Cultural heritage system of turkey on the web, in The ICOMOS \& ISPRS Committee for Documentation of Cultural Heritage CIPA 2007 XXI International Symposium, Proceedings of the XXI International Symposium CIPA 2007 AntiCIPAting the future of the cultural past, ATHENS: National Techical University of Athens printing un, Atene

Pietroni E., Forte M., 2007. A virtual collaborative environment for archaeology through multi-user domain in the web, in The ICOMOS \& ISPRS Committee for Documentation of Cultural Heritage CIPA 2007 XXI International Symposium, Proceedings of the XXI International Symposium CIPA 2007 AntiCIPAting the future of the cultural past. Atene, 01-06 ottobre 2007, National Techical University of Athens printing un., Athene, vol. 1

Zlatanova S., Rahman A., Pilouk M., 2003. 3D GIS: current status and perspectives, Joint International Symposium on Geospatial Theory, Processing and applications, Ottawa

http://www.culturanuova.it/

http://potree.org/ 
http://mrdoob.github.com/three.js/

\section{ACKNOWLEDGEMENTS}

The survey was carried out for Opera Santa Maria Novella, in order to complete the research developed by the Convention entitled "Survey for the knowledge of the Convent of Santa Maria Novella", year 2005, renovated in 2007, entered into between the Department of Architecture, prof. Maria Teresa Bartoli, and the Municipality of Florence, Department of Building and Old Churches.

Special thanks also arch. Massimo Chimenti that he has provided the platform Modus Explorer for viewing highdefinition images. New Culture also has in place an agreement with the department of the University of Genoa DICCA to facilitate the exchange of know-how and the development of new technologies applied to cultural heritage 